\title{
Smart lighting protection skin using QTC pills for aircraft realtime load monitoring
}

\author{
Yoshiro SUZUKI*, Toyoaki SUZUKI*, Akira TODOROKI* and Yoshihiro MIZUTANI* \\ *Tokyo Institute of Technology, \\ 2-12-1 Ookayama, Meguro-ku, Tokyo 152-8552, Japan \\ E-mail: ysuzuki@ginza.mes.titech.ac.jp
}

Received 5 February 2014

\begin{abstract}
For real-time load monitoring, we present a new smart lighting protection skin using resistive touchpad techniques that covers measuring objects with a flexible sheet composed of multiple tiny sensors. We measured loading from off-plate direction, including quasi-static indentation and dynamic impact. We believe our method is suitable for load monitoring of composite aircraft structures. A metal film or mesh coving the fiber composite components of our system acts as both the load sensor and wiring, and a lightning protection shield (LPS). The sheet consists of an upper LPS layer and a lower layer, with multiple pressure-sensitive elastomer pills arranged between layers. The lower layer is a grid consisting of high-electrical-resistance nichrome wires and low-resistance copper wires. The pressure-sensitive pill becomes conductive when compressed. When the sensor sheet is indented, a compressed pill creates a new electrical path between the two layers and the electrical potential equalizes at that point. This event triggers the application of a potential across the nichrome wires that form the $x$ axis, enabling measurement of the $x$ coordinate. The orthogonal potential gradient is then applied to the lower layer so that the $y$ coordinate can be measured. The $x$ and $y$ coordinates are recorded quickly and describe the quasi-static load point with an error of $29 \mathrm{~mm}$ or less. Additionally, we can estimate the peak value of the impact load by measuring the electrical resistance of the pressure-sensitive pill with an error of $17 \%$.
\end{abstract}

Key words : Smart material, Sensor, Composite material

\section{Introduction}

When damage occurs to a structural component from an unexpected load such as impact of something, an indentation load must occur on its surface. It would be helpful if this indentation was detected immediately. Fiber composite components of aircraft are covered with a thin metal mesh or film (usually copper or aluminum) of approximately $0.3 \mathrm{~mm}$ in thickness which provides lightning protection to the aircraft as shown in Fig. 1 (Dexmet corporation, 2014, Kawakami and Feraboli, 2011, Feraboli and Miller, 2009, and Boeing, 2014). Using resistive touchpad techniques (Cok, et al., 2004, Downs, 2005, and Stetson, 2006), we devised an in-situ method for estimating the position and force of the load by inserting pressure-sensitive materials between this lightning protection shield (LPS) and the component. The LPS, in addition to providing protection from lightning, also becomes an integral part of the load sensor circuitry. There have been few studies that discuss the repurposing of LPSs (Suzuki, et al., 2012a, 2012b). Our proposed system is a thin flexible sheet less than $3 \mathrm{~mm}$ thick that can be attached to a curved surface. It requires no special equipment, uses a limited number of DC power supplies and voltage sensing equipment, and can be replaced if broken.

Carbon fiber reinforced plastic (CFRP) exhibits many advantages over metallic material, including corrosion resistance, lower weight, and superior mechanical properties such as specific strength and specific stiffness. CFRP is used as a structural material in commercial aircraft to improve cruising range and fuel efficiency by reducing aircraft weight. For example, the Boeing 787 Dreamliner, which has been in service since November 2011, uses 50\% CFRP by weight. The reliability of structural damage inspections where CFRP is used has become important. 


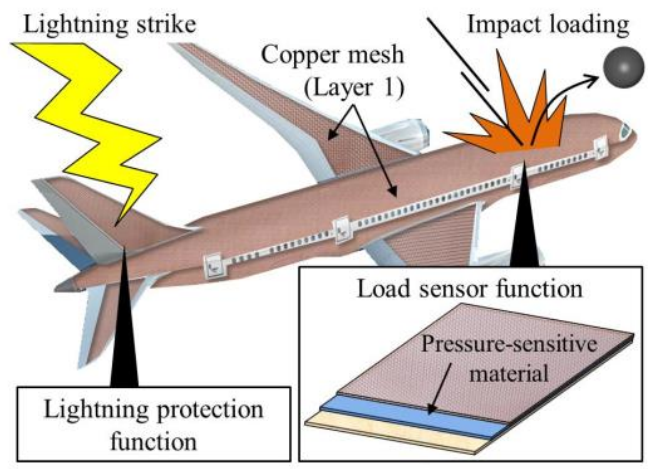

Fig. 1 Schematic of the proposed sensor sheet that has two functions: lightning protection; and load sensor (Boeing, 2014).

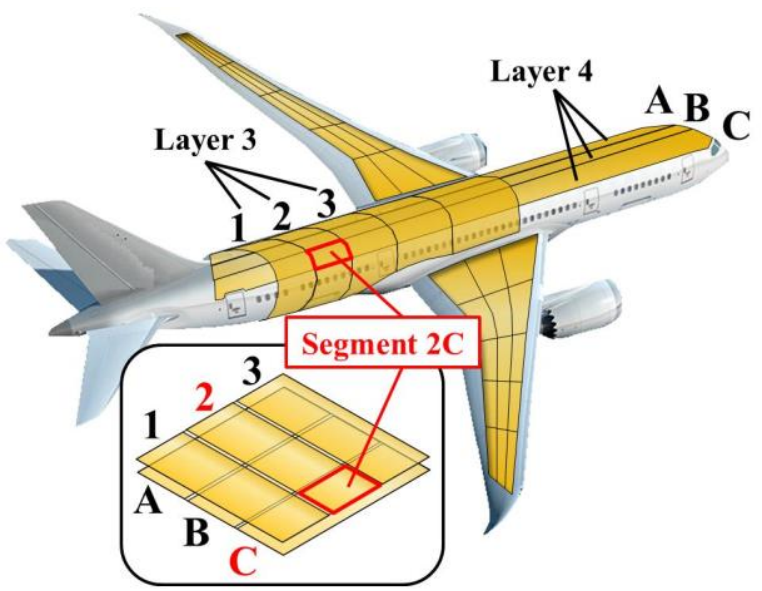

Fig. 2 Schematic of Layers 3 and 4 under Layers 1 and 2 that explains how to specify indented section from the entire structure (Boeing, 2014).

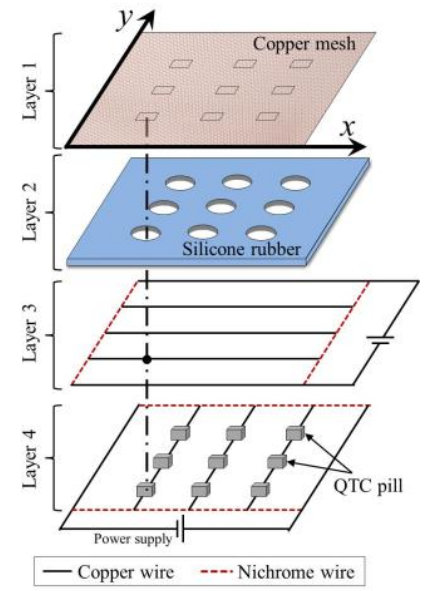

Fig. 3 Magnification of segment 2C that explains how to estimate exact position and value of loading.

Delamination is a serious mode of damage for fiber reinforced plastic (FRP) structures. In laminated FRPs, impact load from off-plate direction can cause layers to separate with significant loss of mechanical strength (Alderson and Evans, 1992 and Kondo, et al., 2007). Delamination in aircraft structures can be caused by low-velocity impacts such as bird strikes, hail, runway debris, or dropped tools. Ultrasonic (Cantwell and Morton, 1985 and Scarponi, 2000) and infrared thermography (Bates, et al., 2000 and Vijayaraghavan, et al., 2010) inspections are used as delamination racking is difficult to detect visually. However, these methods are time-consuming and require taking the aircraft out of service.

The flexible sensor sheet in this study can detect the position and extent of out-of-plane loading in large FRP structures quickly and at low cost. It cannot detect internal damage to the structure of the aircraft, so detailed inspection of the area in question using other methods such as ultrasound or electrical resistance are required (Suzuki, et al., 2012a, 2012b, Suzuki, et al., 2011a, 2011b, and Todoroki, et al., 2005). This localization of inspection can save a significant amount of time. It becomes possible to inspect large structures such as aircraft components rapidly at low cost by dividing the inspection method into two stages: our proposed method, which quickly locates areas of potential damage and a detailed inspection for internal damage is conducted by another method. Inspection of composite aircrafts after every flight would reduce the environmental load and improve flight safety and economic efficiency (lowest possible factor of safety for each component, high fuel efficiency). Details of the proposed multi-functional sheet are presented in Section 2. Detailed experimental procedure and results of the accuracy of position and value experiments of loading are presented in Sections 3, 4, respectively. We conducted both quasi-static indentation and falling weight impact tests for the load identification. 


\section{Principle of load identification \\ 2.1 Configuration of the sensor}

The proposed sensor sheet consists of the following four layers (Figs. 1-3):

- Layer 1 is a flexible copper mesh (Fig. 1), acting as both LPS and signal path between pressure-sensitive materials and LCR meters (electrical resistance measuring instruments).

- Layer 2 is a nonconductive silicone rubber sheet acting as load support and insulator between Layers 1 and 3 (Fig. 3).

- $\quad$ Layer 3 is a grid of nichrome and copper wires (Fig. 3).

- Layer 4 has the same configuration as layer 3 but it is rotated by 90 degrees (Fig. 3).

Quantum tunneling composite (QTC) pressure-sensitive pills, which are silicone pills with multiple nano-sized conductive particles and sharp thorns on these particles, are arranged between layers 3 and 4 at the intersection points of the copper wires (Fig. 3). The QTC pill has been developed for practical application of switching and sensing systems by Peratech Ltd., Darlington, UK (Peratech Ltd., 2014). The thorn surfaces are covered with an insulating film, which allows the conductive particles to get close but not touch. As shown in Fig. 4, when the pill is compressed, the distances between the particles in the pill shorten and conducting paths arise between the particles owing to a tunnel effect. As a result, the electrical resistance of a QTC pill decreases sharply and continuously. The pill transforms from an almost perfect electric insulator to a metal-like conductor. The deformation-resistance curve is smooth and repeatable. Flexible load sensors of arbitrary size and shape can be made of the QTC. Therefore, the QTC pill seems to be suitable for inspection sensing systems for an airplane that suffers sharp fluctuations in temperature because it can be made to withstand extreme temperatures limits (from -60 to $250{ }^{\circ} \mathrm{C}$ ).

\subsection{Procedure for load identification}
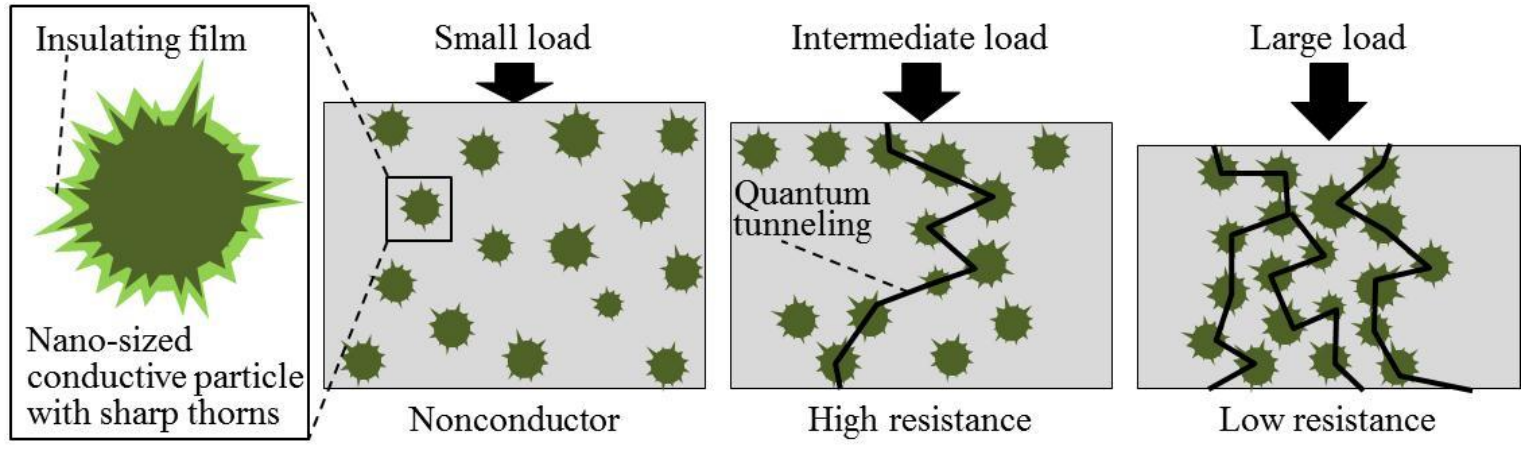

Fig. 4 Schematic of a cross section of the quantum tunneling composite (QTC) pressure-sensitive pill. Black lines show electrical current paths.

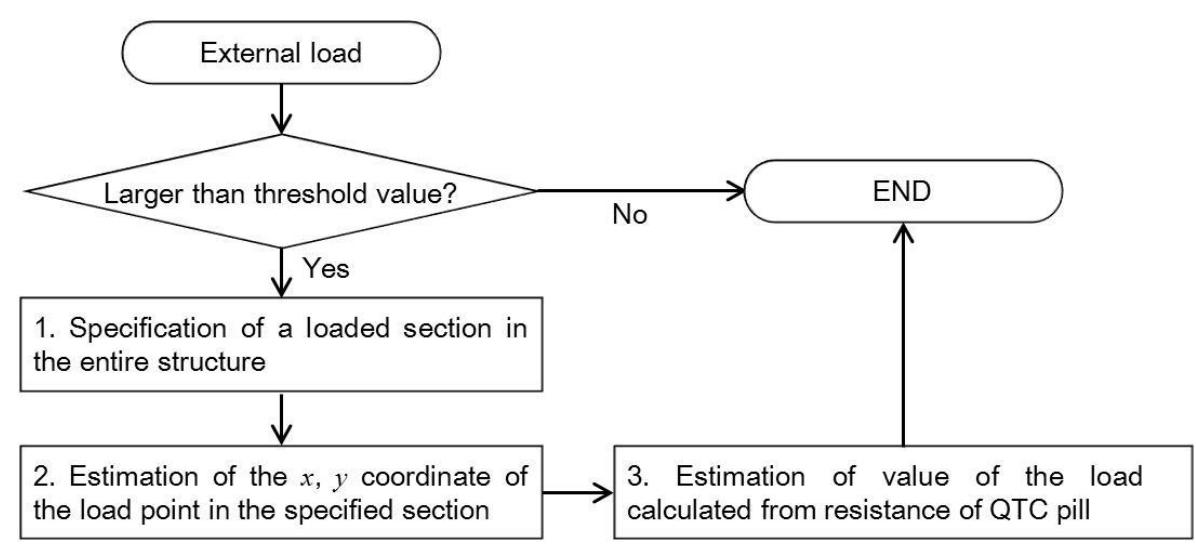

Fig. 5 Flowchart of procedure for the load identification. 
- Copper wire ---. Nichrome wire

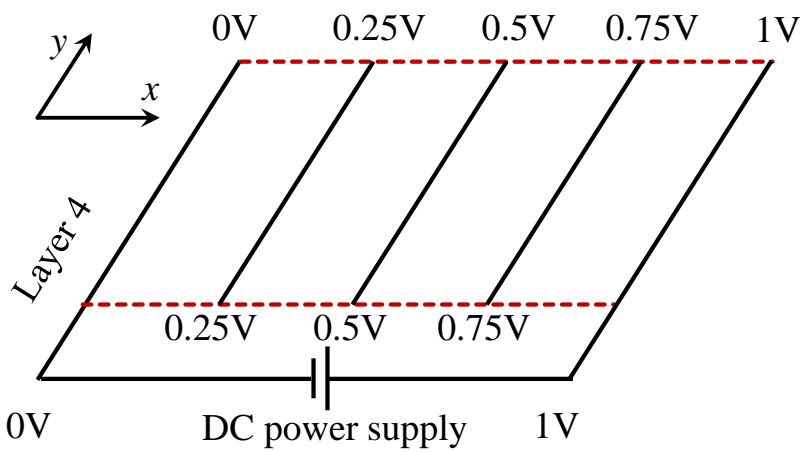

Fig. 6 Potential gradient applied to layer 4 when estimating the x coordinate of load position.

The proposed procedure for load identification is schematically illustrated in the following three steps and Fig. 5:

\subsubsection{Step 1: Specification of a loaded section in the whole structure}

Fig. 2 shows a schematic of layers 3 and 4. These conductive layers, explained in 2.1 above, are arranged in rectangles orthogonal to each other, named 1, 2, 3, etc. and A, B, C, etc. For example, section $2 \mathrm{C}$ occurs where rectangles 2 and $\mathrm{C}$ overlap. When an indentation load is applied to section $2 \mathrm{C}$, rectangle 2 has electrical contact with rectangle $\mathrm{C}$. By applying a unique voltage to each rectangle in layer 4, the voltage of rectangle 2 becomes equal to that of rectangle $\mathrm{C}$, identifying $2 \mathrm{C}$ as the indented section. Current flows between the two rectangles only when a physical load causes the rectangles to make electrical contact with each, otherwise power is not consumed.

\subsubsection{Step 2: Estimation of the exact position of loading after specifying the loaded section}

After identifying the loaded section, a more precise position for the load is estimated using resistive touchpad

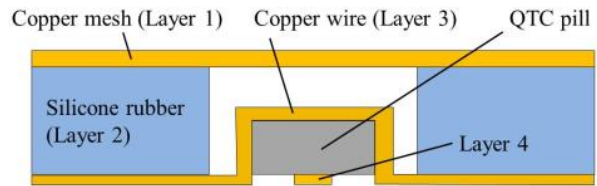

(a)

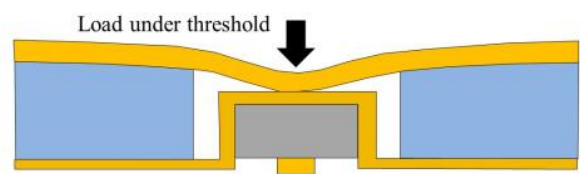

(b)

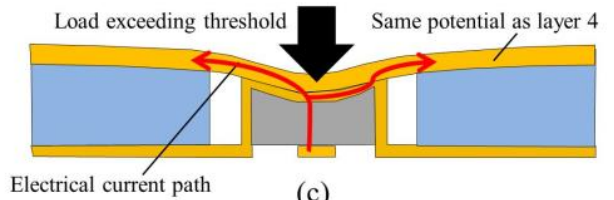

(c)

Fig. 7 Schematic of a cross section of the load sensor. Red arrows show electrical current paths.

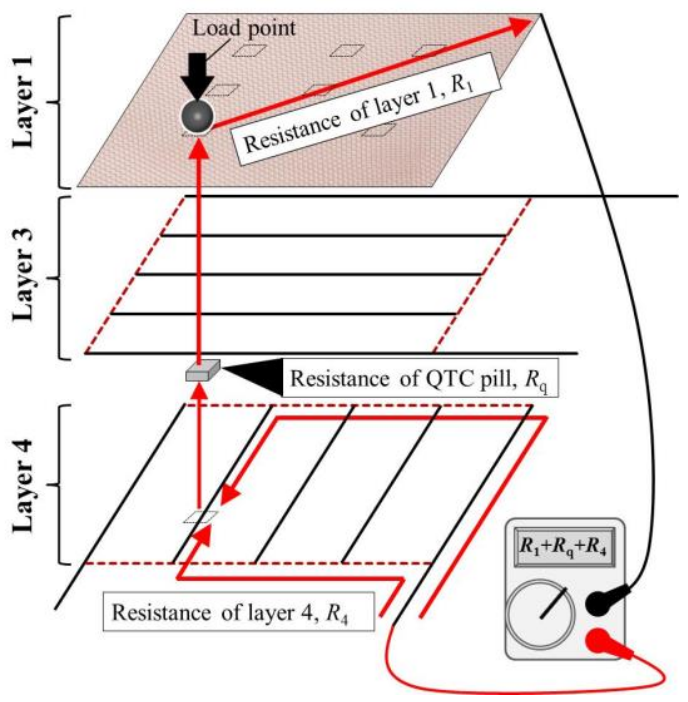

Fig. 8 Estimation of load value calculated from resistance of QTC pill, $R_{\mathrm{q}}$. Measured resistance is $R=R_{1}+R_{\mathrm{q}}+R_{4}$. Red arrows show current path. 
techniques (Cok, et al., 2004, Downs, 2005 and Stetson, 2006). Only the specified section, 2C in this case, needs to be investigated. The task of position estimation is divided into two steps: specification of the loaded section and estimation of the exact position in the specified section. This two-stage method results in a high degree of accuracy over large areas such as those of aircraft components.

Fig. 6 shows the gradient of electrical potential along the $x$ direction when applying a voltage of 1 volt to the two nichrome wires of layer 4 . Voltage drop along the nichrome wires results in a different potential applied to each copper wire, which has equal potential along its length. This occurs because the resistance of nichrome wire is much higher than that of copper. When the sheet is indented, a compressed QTC pill provides a new electrical path between layers 1, 3 , and 4 at the load point. This results in the potential of layer 1 equaling that of layer 3 as shown in Fig. 7. Fig. 7(a) is a no-load condition with no contact between layers 1, 3, and 4; layer 1 has no potential applied. When the sensor is indented, layer 1 bends and touches a copper wire in layer 3. If the load is smaller than the threshold value, the QTC pill under the loading point remains nonconductive as shown in Fig. 7(b). If it exceeds threshold, the pill becomes conductive as shown in Fig. 7(c) and layers 1, 3 and 4 then have the same voltage potential. The $x$ gradient described above can then be measured, resulting in an accurate reading for the $x$ coordinate. Ordinary voltmeters, connected in parallel when measuring, have a high internal resistance and do not affect the reading of a compressed QTC pill, which has a much lower resistance.

Once $x$ has been obtained, layer 4 voltage is removed and a potential difference of 1 volt is applied between both ends of layer 3. Linear potential gradient along the $y$ axis is then measured on layer 1 .

\subsubsection{Step 3: Estimation of value of load}

The schematic diagram for our system, which estimates the value of a load, is shown in Fig. 8. The electrical resistance between layers 1 and 4 is measured. Measured resistance $\left(R_{\mathrm{m}}\right)$ is the sum of the resistance of layer $1\left(R_{1}\right)$, and layer $4\left(R_{4}\right)$ and that of the QTC pill at the load point $\left(R_{\mathrm{q}}\right) . R_{1}$ and $R_{4}$ can be calculated from the position of the load point. Therefore, $R_{\mathrm{q}}$ can be determined from Eq. (1).

$$
R_{\mathrm{q}}=R_{\mathrm{m}}-R_{1}-R_{4}
$$

Because $R_{\mathrm{q}}$ decreases monotonically with increasing load, indentation load can be estimated.

\subsection{Advantages over other inspecting methods}

Compared with other inspecting methods, the advantages of the proposed method are the following:

- There is no need to constantly poll individual sensors across the structure. Load can be measured within a few milliseconds. There is no current flow or power consumption unless a load is applied. The entire structure can be monitored in real-time regardless of its size. These factors result in low cost.

- The LPS performs three roles: lightning protection, load sensor, and wiring. This suppresses structural weight increase and keeps wiring to a minimum.
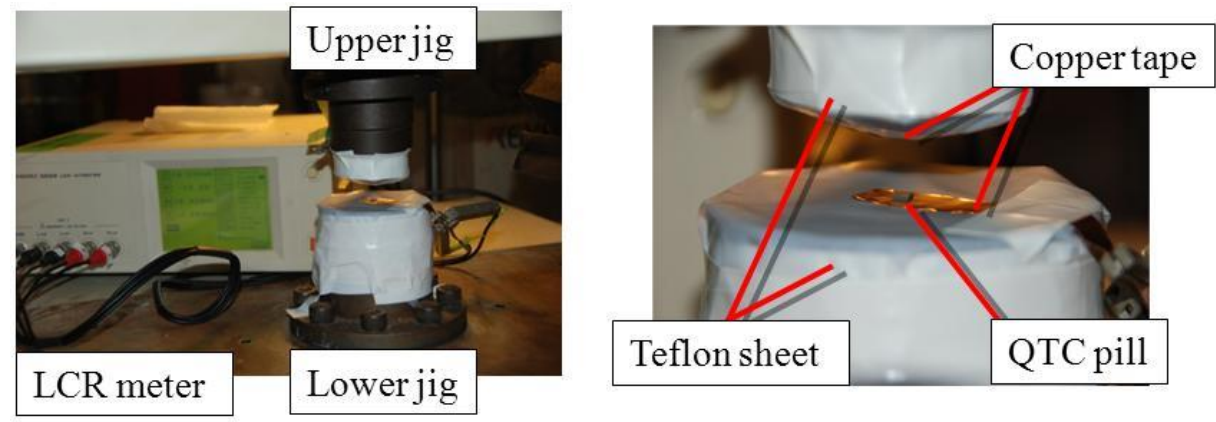

Fig. 9 Experimental setup for measuring deformation-resistance curve of a QTC pill. 
- The sensor sheet is affixed to the structure surface. The mechanical properties of the object being measured are not affected as drilling, or otherwise embedding foreign material under the surface, is not required. Surface mounting makes replacement much easier.

- The proposed sensor can monitor impact and indentation load on a structure regardless of shape or construction material.

\section{Experimental procedure}

This section explains experimental procedure for testing the practical feasibility of the proposed load identification technique. Note that we conducted no experiments under repeated loading because it is unlikely that a load occurs multiple times on the exact same position in a large structure of an airplane.

\subsection{Quasi-static compression test for a QTC pill}

The relationship between resistance and compression deformation of a QTC pill, which is $1.8 \mathrm{~mm} \times 1.8 \mathrm{~mm} \times 1.0$ $\mathrm{mm}$ in size, was examined by conducting a compression test. The schematic of the experimental setup is shown in Fig. 9. The upper and lower jigs were covered with a Teflon sheet for electrical isolation. Copper tape was attached to both jigs, and then a QTC pill was put between them. The pill was compressed by applying a static load using a universal testing machine (Autograph AG-I/100kN, Shimadzu Co., Kyoto, Japan) at the cross-head speed of $0.04 \mathrm{~mm} / \mathrm{min}$. During the test, the electrical resistance between the two copper tapes was measured by a meter (LCR meter, model 3522-50, HIOKI, Nagano, Japan).

\subsection{Quasi-static indentation test for the load identification 3.2.1 Load position}

The practicality of the proposed load identification method was verified. The schematic of the system used for load identification is shown in Fig. 10. The total thickness of the sensor sheet was less than $3 \mathrm{~mm}$. We conducted a quasi-static indentation test with the Autograph. A hemispherical indenter of $15.9 \mathrm{~mm}$ diameter was set on top of the 4-layer sensor sheet just above a QTC. The silicon rubber is twice as thick as the QTC and the distance between the QTC and copper mesh is $1 \mathrm{~mm}$ as shown in the enlarged cross-sectional view of Fig. 10. The diameter of a circular hole of the silicone rubber sheet is $3.5 \mathrm{~mm}$ and much smaller than that of the indenter. Therefore, the silicon rubber around a QTC is compressed to half of the original thickness when an indentation load causes the copper and QTC to make electrical contact with each other, which prevents a low indentation such as regular aerodynamic loading during flight from making the contact. In general, aerodynamic load on commercial aircraft during flight is very small (a few

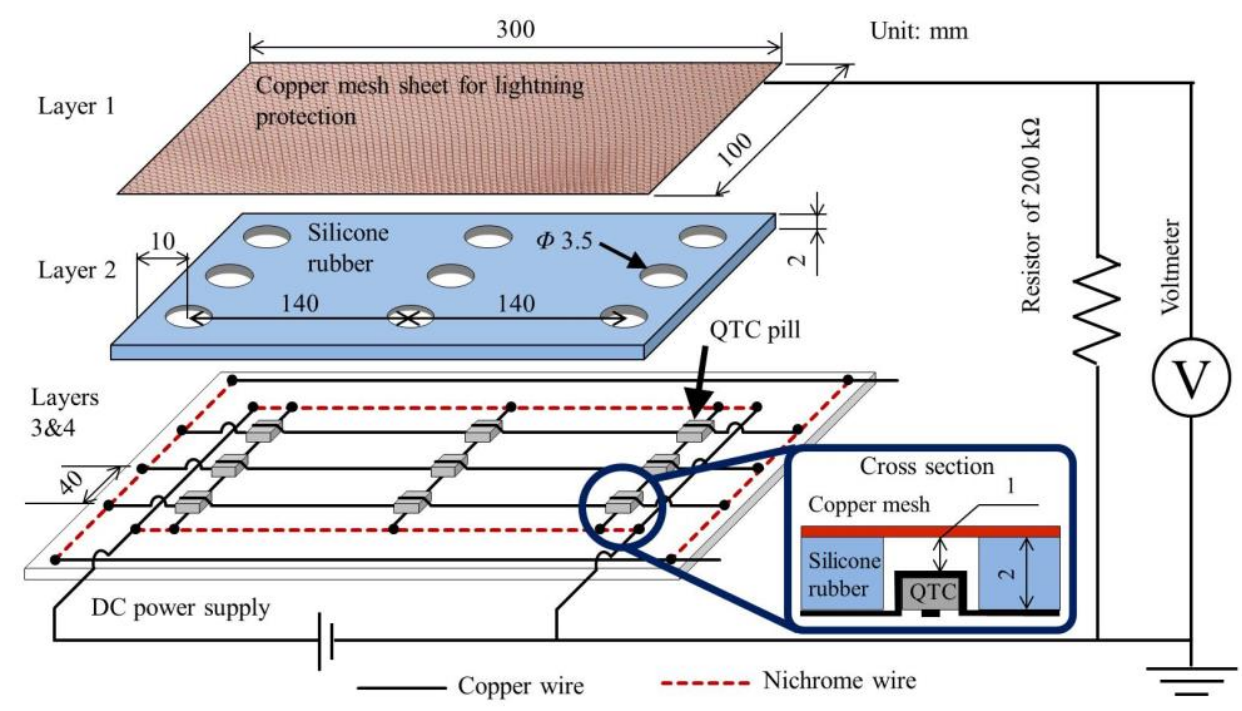

Fig. 10 Schematic and physical layout of the test system used for load identification. 


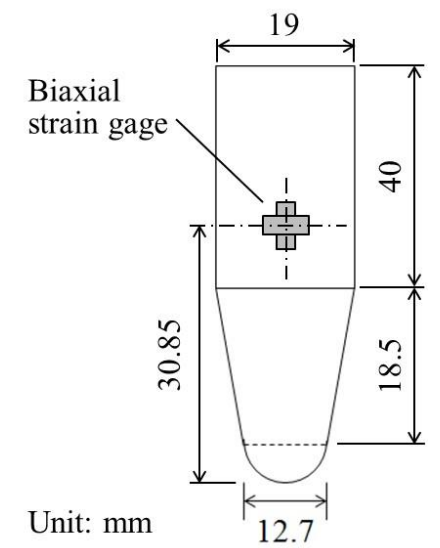

Fig. 11 End part of the impactor and strain gage attached on the surface used for the falling weight impact test.

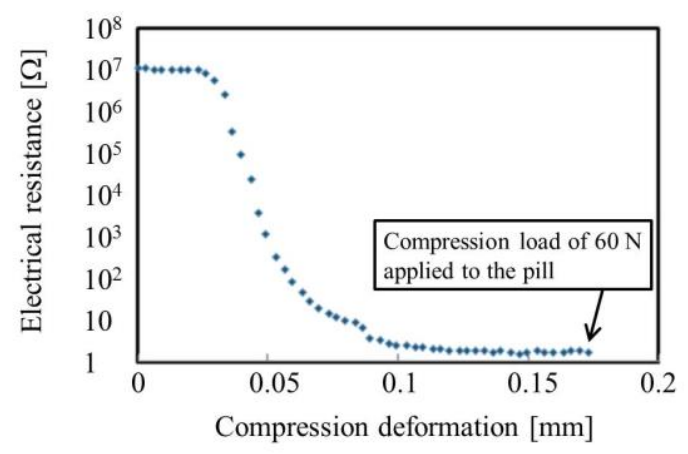

Fig. 12 Measured relationship between compression deformation and resistance of a QTC pill.

newtons). Therefore, adhesion between the silicon rubber sheet and the copper wires would not be easily separated even when the aerodynamic load is repeated. In addition, if necessary, a thin flexible film with embedded copper wires can be inserted under the silicon rubber sheet instead of adhesion (Lin, et al., 2001) in order to enhance the sensor's durability.

Layer 1 voltage potential was measured with an electrometer (PCD-300A, Kyowa Electronic Instruments Co, Ltd, Japan) at a constant static load of $0.4 \mathrm{kN}$ when applying $1 \mathrm{~V}$ across the ends of layer 4 with a stabilized DC power supply (PW18-1.3AT, Kenwood, Japan). It was assumed that voltage drop across a nichrome wire was linearly proportional to the $x$ coordinate. The potential of layer 1 was measured and the $x$ coordinate of the loading point was calculated. The potential of layer 1 was measured again for estimation of the $y$ coordinate after voltage to layer 4 was removed and a voltage of 1 volt was applied across the ends of layer 3. Layer 1 was grounded through a resistor of 200 $\mathrm{k} \Omega$ and the voltage is measured across it. This resistance is so much larger than the compressed QTC pill that voltage drop across the pill does not decrease the estimation accuracy of the load position.

This test was repeated for each of the nine QTC pills in the sensor as shown in Fig. 10. Comparison of the actual and estimated load positions is discussed in the next section.

\subsubsection{Load value}

We proposed to estimate the value of loading applied to the sensor sheet by using a load-resistance curve that is previously obtained. At first, we measured the relationship between the resistance of a QTC pill in the sensor, $R_{\mathrm{q}}$ and the value of the quasi-static indentation load when the sensor sheet was indented with a hemispherical indenter of 15.9 $\mathrm{mm}$ diameter. The indenter was set on the surface of layer 1 (copper mesh) above the central QTC pill and indented at a cross-head speed of $0.05 \mathrm{~mm} / \mathrm{min}$. The resistance between layers 1 and $4, R_{\mathrm{m}}$ was measured by the LCR meter. The resistance of the QTC pill $\left(R_{\mathrm{q}}\right)$ was calculated by substituting $R_{\mathrm{m}}$ into Eq. (1). The value of load was measured by the Autograph.

From the load-resistance curve thus obtained, value of an indentation load can be calculated. To test the practicality of the proposed estimation scheme, a quasi-static indentation load of 0.25 to $0.4 \mathrm{kN}$ was applied to the surface of layer 1 above a QTC pill. During the test, $R_{\mathrm{m}}$ was measured by the LCR meter.

\subsection{Impact test for the load identification}

Falling weight tests were conducted to verify the feasibility of identifying impact loads using the proposed sensor. The test impactor consisted of a long dart with a hemispherically shaped headstock $12.7 \mathrm{~mm}$ in diameter dropped through a guide as shown in Fig. 11. The impact energy was calculated by multiplying the total mass of the impactor by the drop height. A biaxial strain gage (KFG-2-120-D16-16, Kyowa Electronic Instruments Co, Ltd, Japan) was mounted on the impactor surface for estimating value of impact loading. The gage was $30.85 \mathrm{~mm}$ from the impact 
point. The load-strain curve of the gage was previously obtained so that value of loading on the impactor can be estimated with high accuracy using the strain gage resoponse. Consequently, we can compare the proposed sensor response with the strain gage.

\subsubsection{Load position}

The sensor sheet was impacted in the center by dropping a mass of $3.19 \mathrm{~kg}$ from a height of $100 \mathrm{~mm}$ with an impact energy of $3.13 \mathrm{~J}$. The potential of layer 1 as shown in Fig. 10 was measured and only the $y$ coordinate of the loading point was calculated in the same way presented in Section 3.2.1.

\subsubsection{Load value}

The sensor sheet was impacted in the center by dropping a mass of $1.35 \mathrm{~kg}$ from a height of $10 \mathrm{~mm}$ with an impact energy of $0.132 \mathrm{~J}$. If the sensor can detect such a small impact, the practical feasibility is regarded as enough high. The

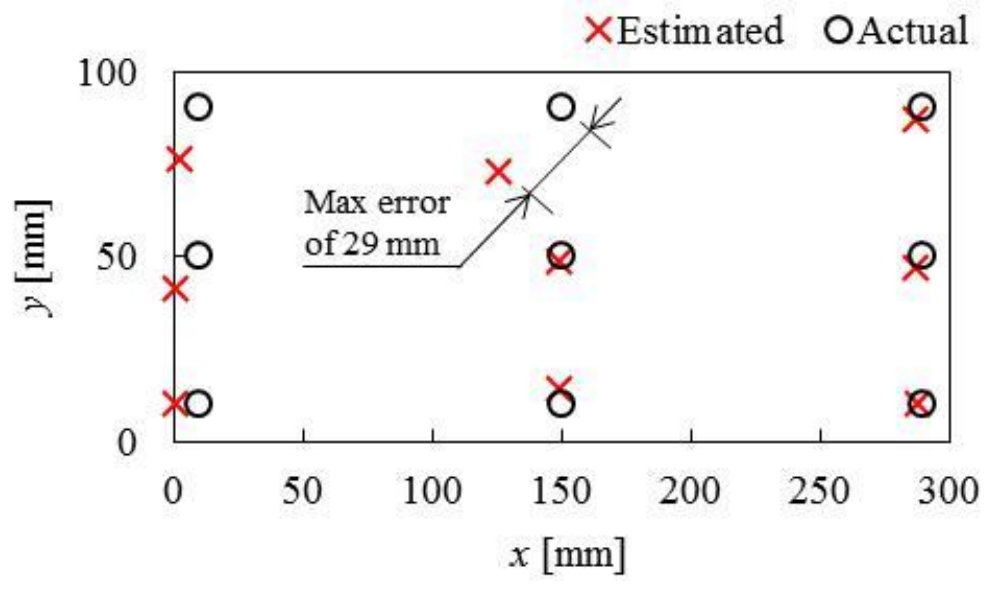

Fig. 13 Actual and estimated position of quasi-static indentation loads.

Table 1 Average and standard deviation of the error between the actual and estimated loading positions.

\begin{tabular}{cccc}
\hline Item & $\begin{array}{c}\text { Distance between actual } \\
\text { and estimated position } \\
{[\mathrm{mm}]}\end{array}$ & $x$ coordinate [mm] & $y$ coordinate [mm] \\
\hline Average error & 9.05 & 5.59 & 6.54 \\
Standard deviation of error & 8.33 & 6.86 & 5.49 \\
\hline
\end{tabular}

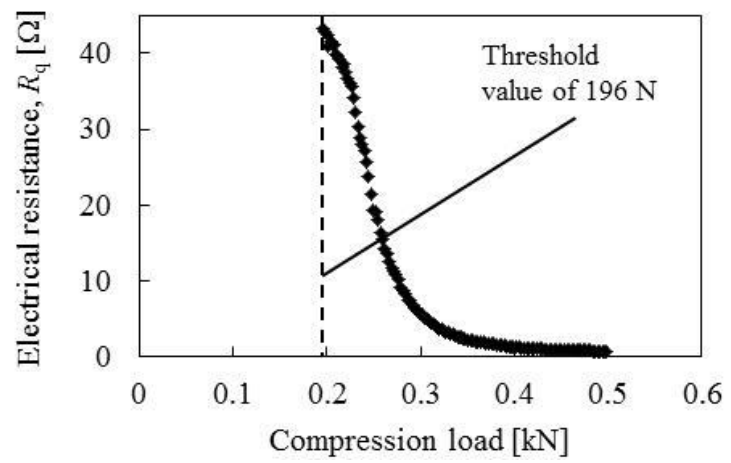

Fig. 14 Relationship between resistance of a QTC pill in the sensor, $R_{\mathrm{q}}$ and value of a quasi-static indentation load.

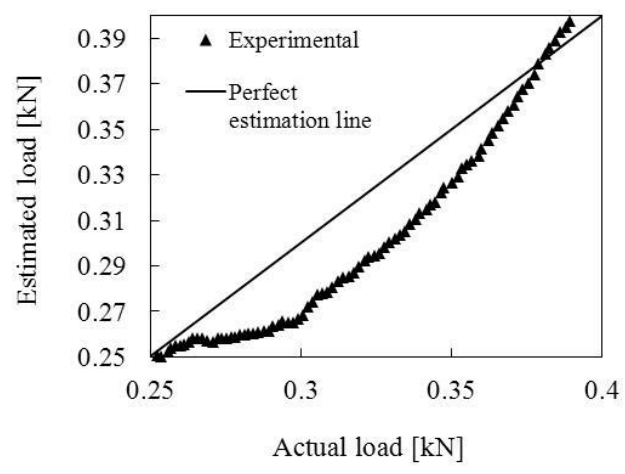

Fig. 15 Comparison of the actual and estimated values of static indentation load. 
resistance of a QTC pill located at the center, $R_{\mathrm{q}}$ was measured and the peak value of loading was estimated in the same way presented in Section 3.2.2.

\section{Characterization and results}

\subsection{Quasi-static compression test for a QTC pill}

The measured resistance-displacement curve of the QTC pill is shown in Fig. 12. The vertical axis is the logarithmic resistance and the horizontal axis is the compression deformation in $\mathrm{mm}$. When a static load of $60 \mathrm{~N}$ was applied to the pill, the compression deformation increased to $0.173 \mathrm{~mm}$ and its resistance fell to $1.66 \Omega$ from approximately $10^{7} \Omega$.

\subsection{Identification of quasi-static loading}

\subsubsection{Load position}

The actual and estimated positions of the quasi-static loads are shown in Fig. 13. Table 1 presents that the average and standard deviation values of the error in distance between the actual and estimated load positions are $9.05 \mathrm{~mm}, 8.33$ $\mathrm{mm}$, respectively. The maximum error occurred when $x=150 \mathrm{~mm}$ and $y=10.0 \mathrm{~mm}$. The estimated load position was $x$ $=174 \mathrm{~mm}$ and $y=26.4 \mathrm{~mm}$; therefore, the resultant error was $29 \mathrm{~mm}$. Such a small error is not prohibitive to practical use because the objective of this process is in identifying the load position (i.e., areas of potential damage) not detecting internal damage in the structure. Another method is used for detailed inspection of damage subsequently. Consequently, the detailed inspection needs to be conducted for only a circular area with a radius of $30 \mathrm{~mm}$ or longer centering at the estimated load position. It is likely that layer 1 did not reach the same potential as the lower layers because electrical contact between layer 1 and copper wires at the load point was not sufficient enough. We believe the imperfect contact resulted in potential drop across the contact resistance between layer 1 and the copper wires. Consequently, the potential of layer 1 was smaller than the accurate value, which made the estimated position coordinates of the load smaller than the actual ones.

\subsubsection{Load value}

Fig. 14 shows the results of the prior experiment which prepared the load-resistance curve for estimation of an indentation load applied to the sensor sheet. The electrical resistance of a QTC pill in the sensor $\left(R_{\mathrm{q}}\right)$ smoothly decreased as the sensor sheet was indented with the hemispherical indenter up to a maximum load of $0.5 \mathrm{kN}$. The threshold value for detection of load was $196 \mathrm{~N}$. In other words, the sensor sheet is designed to detect only >196 N of loading. This is because we should not detect a lower than $196 \mathrm{~N}$ of load (such as aerodynamic loading during flight of approximately a few newtons) that does not cause damage to the structure. Naturally, the threshold value can be easily

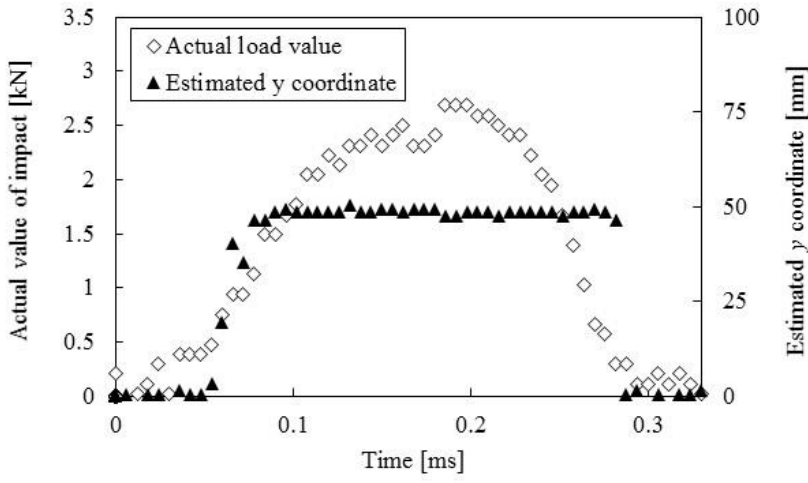

Fig. 16 Results of estimating position of an impact load. Rhombuses show the actual value of loading calculated from the normal strain of the impactor. Triangles present $y$ coordinate of the impact estimated by the proposed sensor.

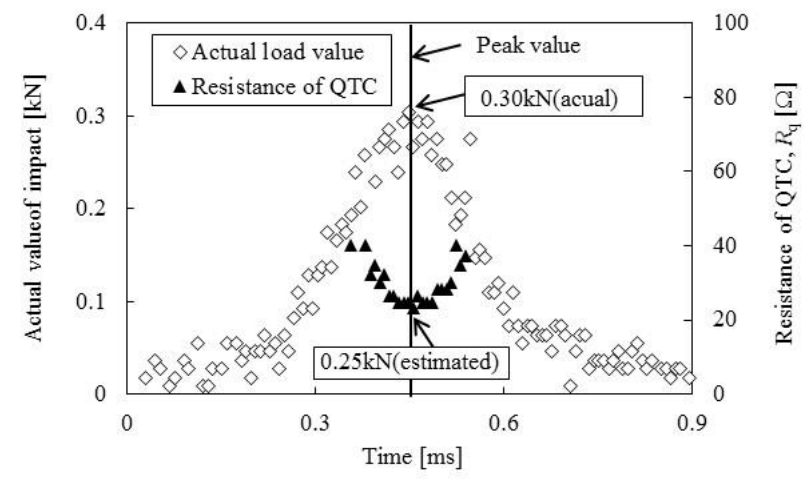

Fig. 17 Results of estimating peak value of an impact load. Rhombuses show the actual value of loading calculated from the normal strain of the impactor. Triangles present resistance of a QTC pill in the sensor, $R_{\mathrm{q}}$. 
changed by using a different thickness of silicon rubber which supports the load applied to the sensor.

Note that the values of the indentation load as shown in Fig. 14 were much higher than those of the compression test for the QTC pill as shown in Fig. 12 and discussed in section 4.1. As a reason for that, the silicon rubber sheet was inserted into the sensor for supporting most of the load applied to the sensor. When the QTC pill in the sensor was compressed, the surrounding rubber sheet was also simultaneously compressed. Conversely, Fig. 12 presents the relation between the electrical resistance and compression deformation of the QTC when a compression load was directly applied to the pill. Therefore, the resistance of the pill quickly decreased to several ohms when applying a low compression load of $60 \mathrm{~N}$.

Fig. 15 presents the relationship between the estimated and actual values of quasi-static indentation load when applying the actual load of 0.25 to $0.4 \mathrm{kN}$ to the sensor sheet. The estimated values were calculated from $R_{\mathrm{q}}$ using the relationship between load and electrical resistance (Fig. 14). The estimated load was between $84 \%$ and $112 \%$ of actual. Again, the maximum error of $16 \%$ is not prohibitive for practical application because the objective of the proposed method is detecting areas of potential damage quickly at low cost. Other subsequent inspections such as ultrasound tests are used to reveal the mode, size, and shape of the damage caused by the detected load.

\subsection{Identification of impact loading 4.3.1. Load position}

Fig. 16 presents the results of estimating position of loading when the sheet was impacted in the center. The rhombuses show the actual value of loading calculated from the strain of the impactor. The triangles present the $y$ coordinate of the impact point estimated by the proposed sensor. The estimated $y$ coordinate started to rise a few ten microseconds behind the strain gage response. Then the $y$ coordinate was kept at a nearly constant value of $49.5 \mathrm{~mm}$ during loading. Since the actual position was $y=50 \mathrm{~mm}$, the error was $0.5 \mathrm{~mm}$.

\subsubsection{Load value}

The results of identifying the value of impact loading are shown in Fig. 17. The rhombuses present the load value and the triangles indicates the resistance of a QTC pill in the sensor, $R_{\mathrm{q}}$. The peak value of loading was calculated from the minimum value of $R_{\mathrm{q}}$ using the load-resistance curve as shown in Fig. 14. Compared to the actual peak value of $0.30 \mathrm{kN}$, the estimated value was $0.25 \mathrm{kN}$ and the error was $17 \%$.

\section{Conclusion}

This paper proposed a new system to measure the position and force of a load using touch-sensitive sensors made up of pressure-sensitive elastomer pills. These sensors could be used to monitor the structural health of composite aircraft structures. A copper mesh sheet used in the system can act both as lightning protection and as part of the wiring of the sensor, leading to overall reductions in weight and thickness (volume) of the entire sensor system. The feasibility of load detection and identification using the proposed method was investigated by static indentation testing. The position of the load was estimated with a maximum error of approximately $29 \mathrm{~mm}$, and the value of the load was estimated with a maximum error less than $16 \%$. These errors are small and will not limit the system in practical use since the objective of the proposed method is in identifying areas of potential damage. Another subsequent method is used for detailed inspection for internal damage. The detailed inspection needs to be conducted only for a circular area with a radius of $30 \mathrm{~mm}$ or longer centering at the estimated load position. As the result of the quasi-static indentation test, the threshold value for detecting a load applied to the sensor was $196 \mathrm{~N}$. In other words, the sensor sheet is designed not to detect a lower than $196 \mathrm{~N}$ of load (such as aerodynamic loading during flight of approximately a few newtons) because this low force does not cause damage to the structure.

Additionally, we conducted falling weight impact tests and revealed that the sensor can estimate the peak value of the impact load by the proposed sensor with an error of $17 \%$. The error of estimating position of the impact was 0.5 $\mathrm{mm}$. 


\section{References}

Alderson, K. L. and Evans, K. E., Low velocity transverse impact of filament-wound pipes: part 1. Damage due to static and impact loads, Composite Structures, Vol. 20 (1992), pp. 37-45.

Bates, D., Smith, G. and Lu, D., Hewitt J., Rapid thermal non-destructive testing of aircraft components, Composites Part B, Vol. 31 (2000), pp. 175-185.

Boeing Co., Homepage: advanced composite use, available from <http://www.newairplane.com/787/design_highlights/\#/visionary-design/composites/advanced-composite-use>, (accessed on 10 July, 2014).

Cantwell, W. and Morton, J., Detection of impact damage in CFRP laminates, Composite Structures, Vol. 3 (1985), pp. 241257.

Cok, R. S., Bourdelais, R. R. and Kaminsky, C. J., Flexible resistive touch screen, US Patent, 0212599 A1, (2004).

Dexmet corporation, Lightning strike protection for carbon fiber aircraft, Advancement of Materials Process Engineering (SAMPE) Conference (online), available from <http://www.dexmet.com/1_pdf/LSP\%20for\%20Carbon\%20Fiber\%20Aircraft.pdf>, (accessed on 10 July, 2014).

Downs, R., Using resistive touch screens for human/machine interface, Analog Applications Journal, 3Q (2005), pp. 5-9.

Feraboli, P. and Miller, M., Damage resistance and tolerance of carbon/epoxy composite coupons subjected to simulated lightning strike, Composites: Part A, Vol. 40 (2009), pp. 954-967.

Kawakami, H. and Feraboli, P., Lightning strike damage resistance and tolerance of scarf-repaired mesh-protected carbon fiber composites, Composites: Part A, Vol. 42 (2011), pp. 1247-1262.

Kondo, H., Aoki, Y., Hiraoka, K. and Hatta, H., Residual indentation, delamination area and CAI strength of CFRP laminates under low-velocity impact, Proceedings of 16th International Conference on Composite Materials, (2007), pp. 1-7.

Lin, M., Qing, X., Kumar, A. and Beard, S. J., Smart layer and smart suitcase for structural health monitoring applications, Smart Structures and Materials 2001: Industrial and Commercial Applications of Smart Structures Technologies, Vol. 98 (2001).

Peratech Ltd., Integration guide, available from <http://www.peratech.com/qtc-science.html>, (accessed on $10 \mathrm{July,} \mathrm{2014).}$

Scarponi, C., Ultrasonic technique for the evaluation of delaminations on CFRP, GFRP, KFRP composite materials, Composites Part B, Vol. 31 (2000), pp. 237-243.

Stetson, J., Analog resistive touch panels and sunlight readability, Journal of the Society for Information Display, Vol. 22 (2006), pp. 26-30.

Suzuki, Y., Todoroki, A., Matsuzaki, R. and Mizutani, Y., Impact-damage visualization in CFRP by resistive heating: development of a new detection method for indentations caused by impact loads, Composites Part A, Vol. 43(2012a), pp. 53-64.

Suzuki, Y., Todoroki, A., Matsuzaki, R. and Mizutani, Y., Indentation-damage visualization in CFRP by resistive heating: analytical verification of the inspection of aircraft using its lightning protection systems, Journal of Solid Mechanics and Materials Engineering, Vol. 6 (2012b), pp. 213-226.

Suzuki, Y., Todoroki, A., Mizutani, Y. and Matsuzaki, R., Impact damage detection in CFRP using statistical analysis of resistance-temperature characteristics, Journal of Solid Mechanics and Materials Engineering, Vol. 5, No. 1(2011a), pp. $33-43$.

Suzuki, Y., Todoroki, A., Mizutani, Y. and Matsuzaki, R., Damage detection in CFRP without data from intact structures using statistical analysis of resistance-temperature characteristics, Transactions of the Japan Society of Mechanical Engineers, Series A, Vol. 77, No. 773(2011b), pp. 181-189 (in Japanese).

Takahashi, K., Park, J. S. and Hahn, H. T., An addressable conducting network for autonomic structural health management of composite structures, Smart Materials and Structures, Vol. 19 (2010), pp. 1-10.

Todoroki, A., Tanaka, M. and Shimamura, Y., Electrical resistance change method for monitoring delaminations of CFRP laminates: effect of spacing between electrodes, Composite Science and Technology, Vol. 65(2005), pp. 37-46.

Vijayaraghavan, G. K., Majumder, M. C. and Ramachandran, K. P., Quantitative Analysis of delaminations in GRP pipes using thermal NDTE technique, Journal of Advanced Manufacturing Technology, Vol. 1 (2010), pp. 60-68. 